\title{
LAS HABILIDADES SOCIOEMOCIONALES EN LOS JÓVENES: UNA PROPUESTA DE DESARROLLO HUMANO INTEGRAL
}

\section{THE SOCIO-EMOTIONAL SKILLS IN YOUNG PEOPLE: A PROPOSAL FOR INTEGRAL HUMAN DEVELOPMENT}

\author{
Lessy Gabriela Jakiwara Grández ${ }^{1}$. Universidad Católica San Antonio de Murcia. \\ España. \\ lessygaby@hotmail.com
}

\section{RESUMEN}

Las habilidades socio-emocionales es un tema de gran importancia que tiene incidencia directa sobre nuestro bienestar y salud; de allí la importancia de este tema y más aún entre los jóvenes que están en una etapa evolutiva en la que las decisiones que tomen y las experiencias que vivan marcan de manera indeleble el cerebro de los mismos y esto a su vez se refleja en las actitudes y comportamiento. Este trabajo trata sobre lo que se ha escrito acerca de habilidades sociales y gestión emocional teniendo en cuenta autores muy conocidos y otros investigadores no tan conocidos no por ello menos importantes.

PALABRAS CLAVE: jóvenes, habilidades sociales, habilidades emocionales, desarrollo humano, pensamiento positivo, neuroplasticidad, bienestar.

\section{ABSTRACT}

Socio-emotional skills is a matter of great importance which has direct impact on our well-being and health; Hence the importance of this subject and even more among the young people who are in an evolutionary stage in which decisions taken and experiences living mark indelibly the same brain and this in turn is reflected in attitudes and behavior. This work is about what has been written about social skills and emotional management take account of well-known authors and others not so well known no less important.

KEY WORDS: young people, skills social, emotional skills, human development, positive thinking, neuroplasticity, wellness.

\section{Cómo citar el artículo:}

Jakiwara Grández L. G. (2016). Las habilidades socioemocionales en los jóvenes: una propuesta de desarrollo humano integral. Revista de Ciencias de la Comunicación e Información, 21(2), 67-80.

doi: http://doi.org/10.35742/rcci.2016.21(2).67-80

\footnotetext{
${ }^{1}$ Lessy Gabriela Jakiwara Grández: Investigadora colaboradora del grupo de investigación sobre "Desarrollo Personal” de la Universidad Católica "San Antonio" de Murcia. Murcia, España.
} 


\section{INTRODUCCIÓN}

Las habilidades socio-emocionales de los jóvenes es el tema que nos ocupa en este trabajo teniendo en cuenta la etapa madurativa de los mismos y se apoya en los conocimientos de investigaciones aportados por científicos que han tenido impacto en los últimos años, sobre todo por los avances aportados por la neurociencia y los beneficios que traería la aplicación de los mismos en el bienestar y la salud.

Tratamos de manera breve los aportes realizados por diversos autores y que nos han permitido establecer relaciones entre las habilidades socio-emocionales y el cerebro y su importancia en la vida social y en la salud psicofísica.

\section{OBJETIVO}

El objetivo principal de este trabajo fue conocer acerca del estado actual de las investigaciones sobre las habilidades socio-emocionales en los jóvenes, en tanto la juventud es una etapa vital muy importante para el desarrollo de las personas debido a que en ella se decide gran parte del futuro de los jóvenes y se puede incidir más en el desarrollo de habilidades socio-emocionales para que logren un desarrollo humano integral.

Hemos revisado bibliografía sobre el tema de autores relevantes como Daniel Goleman, Joe Dispenza, Paul Ekman entre los más relevantes y también hemos revisado alguna revista especializada sobre estos temas tratando de enfocar el mismo desde una perspectiva integral que tome en cuenta los diversos aspectos de las cuestiones socio-emocionales y el enfoque del ser humano como una realidad bio-psico-espiritual.

\section{METODOLOGÍA}

\subsection{Las emociones}

Las emociones son las respuestas automáticas que tenemos los seres humanos ante los diversos estímulos que el entorno nos presenta y en un primer momento tiene que ver con la cuestión adaptativa pues el miedo que es una emoción que nos protege de los peligros y nos ayudan a mantener la supervivencia. Todas las personas sentimos las emociones de una manera única. Las emociones tanto positivas como negativas son contagiosas; de allí su incidencia social.

Las emociones se expresan en el cuerpo y a través del cuerpo de una manera inconsciente: es una respuesta automática por eso es prácticamente imposible mentir acerca de las emociones que vivimos porque nuestro cuerpo nos delata a través de la voz, la expresión facial, la postura corporal, la sudoración y el movimiento corporal.

Las emociones provocan determinados cambios en el cerebro, en el sistema nervioso autónomo que regula el ritmo cardíaco, la respiración y la sudoración y nos preparan para diversas acciones que tienen que ver con la conservación de nuestra integridad física. 
Como señala Ekamn (2003) cada persona tiene sus propios desencadenantes emocionales que son producto de sus experiencias personales y son aprendidas pero también tienen una base genética e histórica como especie humana. En ellas se utilizan músculos faciales para emociones concretas que son universales.

La emocionalidad y la sociabilidad humana están muy ligadas porque cumplen funciones básicas en la lucha por la supervivencia y tiene sus antecedentes en los primates que establecían relaciones de cooperación y conflicto por la comida y el espacio.

\subsection{El sistema de alerta emocional}

Según Paul Ekman (2003) todos los seres humanos tenemos un sistema de alerta emocional que es como un sistema de base de datos abierta que activamos para protegernos de los peligros, de las experiencias que nos han hecho daño, es una forma de proteger la supervivencia. En el comportamiento emocional están implicadas varias áreas del cerebro especialmente la amígdala y el lóbulo frontal y nuestras experiencias personales. Tenemos evaluadores automáticos que son pensamientos automáticos que nos sirven para ahorrar tiempo en circunstancias peligrosas en nuestra vida y que puede ser crucial para nuestra supervivencia, es decir, actuamos sin que nosotros pensemos conscientemente e incluso seamos conscientes del peligro que nos amenaza.

Podemos reinterpretar episodios traumáticos de nuestra vida y curar heridas emocionales al respecto Paul Ekman señala que:

El recuerdo de episodios emocionales, de ésos que elegimos traer a la memoria y que nos provocan que de inmediato volvamos a experimentar las emociones vividas en la situación original, nos brindan la oportunidad de aprender a reinterpretar lo que esté sucediendo ahora en nuestra vida de forma que tengamos la posibilidad de cambiar lo que esté haciéndonos reaccionar emocionalmente (Ekman, 2003, p. 54)

Esto nos sirve como una forma de curación emocional que afectan nuestro bienestar y nuestra salud. Cuando cambia nuestra percepción cambian nuestras emociones, descubrimiento importante porque permite una curación desde este cambio que se ocupa principalmente la psicología cognitiva; "el simple hecho de revisar, con alguien que nos ayude a contemplarlo desde una perspectiva levemente diferente, un recuerdo doloroso puede según Le Doux, contribuir a aliviar gradualmente parte de la ansiedad provocada por el recuerdo perturbador". (Goleman, 206, pp. 114-115).

Las personas que se bloquean emocionalmente tienen sus habilidades cognitivas y sociales igualmente bloqueadas debido a que estas áreas están estrechamente relacionadas.

El "reflejo de sobresalto" es un predictor de que la persona que lo experimenta tiene tendencia a sufrir emociones negativas como el miedo, la ira y la tristeza. La ira expresa hostilidad y genera enfermedades coronarias: eleva la presión sanguínea y aumenta las posibilidades de sufrir un ataque al corazón. 
Las emociones se producen por los pensamientos, los recuerdos y por lo que vemos en nuestra vida cotidiana, incluyendo lo que vemos a través de la televisión, el cine; es decir, las emociones se producen por las experiencias cotidianas que vivimos.

La televisión es uno de los medios de comunicación de masas de mayor consumo porque es barato y accesible; está constantemente emitiendo imágenes que tienen la capacidad de impresionar y afectar nuestra sensación de bienestar y/o malestar emocional a través de asociaciones de pensamiento automático. Diversos investigadores entre ellos Shapiro (2000) ha señalado que "los medios de comunicación ofrecen modelos sociales de conducta inadecuados y los padres corren el peligro de perder parte de su influencia como modelos, y muchas veces tienen la preocupación de controlar lo que ven sus hijos." Además: "La influencia social que ejercen los modelos a través de las películas también parece estar en función del grado de excitación emocional de los observadores...." (Bandura \& Walters, 1988, p. 92). Al respecto:

...las instrucciones de los padres a los hijos sobre cómo tienen que portarse influyen quizá mucho menos en su conducta social que los medios de comunicación audiovisual de masas, a menos que los padres exhiban, como modelos, una conducta en consonancia con las instrucciones que dan (Bandura \& Walters, 1988, p. 59).

Esto nos habla de la importancia de la coherencia de vida en el modelado de la conducta de los niños y jóvenes y la responsabilidad que tenemos los adultos y los padres de familia al igual que los profesores como modelos en la vida de los más jóvenes de la sociedad.

\subsection{Sentidos percepción y emociones}

Captamos la realidad a través de nuestros sentidos, a partir de ellos aprendemos y percibimos la realidad de nuestro entorno. A partir de pensamientos conscientes podemos cambiar nuestra mentalidad y "reinventarnos" para desarrollarnos integralmente y de esta manera crecer y evolucionar como personas. Esta capacidad se va desarrollando desde el nacimiento y sobre todo en el primer año de vida de las personas en las que debemos lograr dos retos: la capacidad de autorregulación emocional que se refiere a la capacidad de sentirse tranquilo y relajado en el entorno y la capacidad de interesarse por el mundo que le rodea y esto lo logra a través de sus sentidos y de la estimulación del entorno que le rodea (Greenspan \& Thordike, 1997). Los citados autores nos señalan que: "La capacidad en la propia regulación es, como se puede observar, una capacidad fundamental que constituye la base real de la experiencia emocional humana. Y esta capacidad de auto organización y regulación se aprende en la más tierna infancia." (Greenspan \& Thordike, 1997, p. 33).

Los lóbulos frontales son fundamentales para la regulación emocional; en el lóbulo parietal se unifican las representaciones procedentes de todos sentidos (vista, oído y tacto) y juegan un papel fundamental en las representaciones mentales según Richard Davidson. La disfunción de los lóbulos frontales puede estar ligada a las emociones destructivas, pues es la zona que se ocupa de la regulación de las emociones. Las emociones destructivas además según Goleman (2003, p:374) 
"parecen interferir con la capacidad de percibir y comprender la información, lo que explica que los niños perturbados presenten problemas de aprendizaje", es decir, las emociones positivas contribuyen a un mejor rendimiento académico en los niños y viceversa, es decir, los niños con problemas emocionales y de socialización tienen dificultades de aprendizaje porque los aspectos emocionales y cognitivos están muy ligados en el cerebro.

Las emociones negativas influyen negativamente en nuestra salud afectando principalmente nuestro sistema inmunitario, las arterias, el corazón, las vísceras y por tanto nuestra vitalidad (Alonso, 2010).

Nuestro cerebro tiene un enorme potencial y nuestros pensamientos lo están modelando:

Lo sorprendente de nuestro cerebro y de nuestro lóbulo frontal es que tienen la capacidad de hacer que un pensamiento se convierta en la única cosa real para nosotros. Gracias al tamaño de nuestra corteza prefrontal, los seres humanos tenemos el privilegio de convertir un pensamiento en algo más real y más importante que cualquier otra cosa. La naturaleza nos ha estructurado para ser así. Cuando convertimos nuestros pensamientos en lo único real y les prestamos atención como si lo fueran, aunamos las funciones primarias del lóbulo frontal para crear una fuerza tan poderosa como cualquier otra cosa en el universo. (Dispenza, 2010, p. 462).

Según Goleman (2003), para liberarnos de una forma errada de percibir las cosas no solamente necesitamos serenidad y meditación sino también liberarnos de una forma errónea de percibir y conocer la realidad.

\subsection{Habilidades sociales: Las neuronas espejo}

Hay una estrecha relación entre Percepción-pensamiento-emoción-conducta-salud y bienestar. Todas las emociones son sociales porque se entablan en la relación con los demás.

Las habilidades sociales nos permiten conectar con los demás. Esta capacidad de conectar con los demás reside fundamentalmente en las neuronas espejo descubiertas por el equipo de investigación del Dr. Giacomo Rizzolatti en la Universidad de Parma en 1992. Gracias al desarrollo de estas neuronas tenemos la capacidad de contagio emocional, empatía y capacidad de aprendizaje por imitación. Se activan desde temprana edad gracias a la interacción entre el bebé, su madre y el entorno social. La desconexión emocional y social con el bebé ocasiona graves problemas en el desarrollo armónico de la personalidad del niño.

\subsection{Habilidades sociales y salud}

La falta de habilidades sociales produce "dolor" psicológico y se experimenta en la misma área del cerebro donde se experimenta el dolor físico pues implica exclusión del grupo, que en épocas antiguas podía significar la muerte; al ser humano esto le produce dolor porque es gregario por naturaleza, es decir, es un ser eminentemente social. Las personas con una red de apoyo emocional tienen mejor salud. 
El cerebro social se encarga de organizar nuestras interacciones sociales, nos conecta con los demás cada vez que nos relacionamos.

La interacción social promueve asimismo la protección de las habilidades mentales. Shari Bassuk y sus colaboradores, de la Escuela de Salud Pública de Harvard, en Boston, comprobaron dicha hipótesis a finales de los años noventa del siglo $X X$. Estos científicos investigaron la influencia de la vinculación social de las personas mayores en su rendimiento congnitivo a lo largo de 12 años. (Nagel, 2013, en "Vejez Personal", Cuadernos Mente y Cerebro $N^{\circ} 5$, p. 59).

La psicología utiliza la palabra "empatía" en tres sentidos actualmente: conocer lo que siente la otra persona, sentir lo que está sintiendo y responder con compasión a lo que le afecta (Goleman, 2006). Ella vincula lo que sentimos con lo que sienten las otras personas de nuestro alrededor; pero ella se logra cuando se tiene una sana autoestima.

\subsection{La inteligencia social}

El cerebro humano está diseñado para conectar y relacionarse con los demás y estas interacciones sociales si son positivas afectan positivamente la salud y si son interacciones tóxicas la afectan negativamente. Al respecto:

El descubrimiento científico más importante de la neurociencia es que nuestro sistema nervioso está programado para conectar con los demás, ya que el mismo diseño del cerebro nos torna sociables y establece inexorablemente un vínculo intercerebral con las personas con la que nos relacionamos. Ese puente neuronal nos deja a merced del afecto que los demás provocan en nuestro cerebro y, a través de él, en nuestro cuerpo y viceversa. (Goleman, 2006, p. 15).

Existen relaciones tóxicas que nos hacen daño a la salud pues si tenemos relaciones conflictivas en nuestro entorno más próximo generamos tasas de hormonas que generan estrés que no ayudan a nuestro sistema inmunitario; estas relaciones son un factor de riesgo de enfermedad y muerte tan importante como el tabaco, la presión arterial alta, el colesterol, la obesidad y el sedentarismo; antes "la ciencia había soslayado el estudio de los caminos neuronales de los problemas de relación, que ha acabado convirtiéndose en uno de los principales focos de interés de la nueva neurociencia social" (Goleman, 2006, p. 22).

Según Goleman (2006) la inteligencia engloba dos grandes bloques que se dividen en conciencia social y aptitud social.

a) La conciencia social es la capacidad de captar los pensamientos y los sentimientos de las personas con las que entramos en contacto. $Y$ son parte de ella:

1. La empatía primordial. Es la capacidad de detectar las emociones ajenas en las relaciones sociales.

2. La sintonía. Que es la capacidad de generar conexión que se expresa en la escucha activa. 
3. La exactitud empática. Es la capacidad de comprender los pensamientos, sentimientos e intenciones de los demás, se asienta en la empatía primordial.

4. La cognición social. Es la capacidad de conocer el funcionamiento del mundo social, de las relaciones sociales y la capacidad para entablar relaciones interpersonales, ella depende de nuestras creencias y pensamientos. Saber comportarse e interactuar con los demás conociendo las normas sociales constituye una medida de inteligencia social.

b) Por otra parte la aptitud social es la capacidad de conseguir éxito en las relaciones cotidianas y forma parte de ella:

1. La sincronía. Consiste en relacionarse bien a nivel no verbal con las personas para tener una adecuada interacción social, es moverse adecuadamente en las interacciones con las demás personas. Los que no consiguen esta capacidad sufren de "disemia" que es la incapacidad de interpretar los signos no verbales que facilitan las relaciones sociales; por ejemplo cuando se puede hablar y de que se puede hablar en una conversación, también incluye en este problema no respetar las distancias interpersonales de relación; en general implica una conducta "no conectada" con lo que ocurre a su alrededor, es una forma de "ceguera social", las personas sean niños o adultos que padecen disemia son aislados socialmente, eso dificulta el encuentro y el crecimiento personal e implica dolor. Esta es una cualidad entrenable y se puede mejorar la sincronía que beneficia las relaciones sociales en los que se enseña a leer los gestos, las posturas, el contacto físico, el tono de voz, el contacto ocular y el ritmo de las conversaciones. Siedel y Kannin, comparten la tesis de que las competencias sociales tienen una base genética pero pueden educarse.

2. La presentación de uno mismo. Es la capacidad de controlar y encubrir las emociones (que se refiere sobre todo al autocontrol); las personas diestras en esta cualidad demuestran seguridad en sí mismo e impactan positivamente a las personas de su entorno.

3. La influencia. Es el uso del tacto y la serenidad cuando se ejerce autoridad.

4. El interés por los demás. Es la compasión y la empatía que nos inspiran las demás personas.

El modelo de Daniel Goleman tiene sus antecedentes en el modelo de Karl-LudwingHoltz que en la década del 90 del siglo pasado señalaba que la estructura de la inteligencia social incluía las siguientes capacidades:

1. Expresión. Que es la exteriorización de conocimientos, sentimientos y deseos.

2. Recepción. Que es la capacidad de oír a los demás y entender sus intenciones y motivaciones.

3. Apertura. Aceptación de los argumentos ajenos y las críticas.

4. Cooperación. Actuar en equipo y atender las necesidades de los demás.

5. Conformación. Establecer, cuidar y mantener las relaciones sociales y hablar de acuerdo a los temas que se tratan en las conversaciones y en las situaciones sociales.

6. Identificación. Atender los propios objetivos y limitaciones. 
Martín Süss, Kristin Seidel y Susanne Weis han trabajado un método para medir la inteligencia social:

No requiere ningún cuestionario en el que las personas participantes se valoren a sí mismas o expresen sus conocimientos sobre convenciones sociales. La prueba consiste en ejercicios prácticos que se han de realizar a contrarreloj. Se trata de un test de rendimiento.

Según Süss, la inteligencia social consta de tres componentes, al menos: percepción social, comprensión social y memoria social. Para cuantificar cada uno de estos componentes se dispone de textos, material sonoro, imágenes y vídeos que han de ser valorados por los probandos. Las personas con una buena percepción social se caracterizan por captar con exactitud los sentimientos de otros al ver en fotografías las expresiones de sus caras. Les basta contemplar fotogramas de una película para reconocer las relaciones entre las personas que en ella aparecen.

La memoria social se valora, entre otros métodos mostrando instantáneas de diversas parejas. Luego se muestran imágenes individuales que se han de emparejar bien. Otra prueba consiste en oír una breve conversación entre dos personas, en la que se incluya información de interés social. Luego hay que recordar detalles del diálogo, capacidad que, por otra parte, se pone a prueba en cualquier reunión de sociedad. (Marschall, 2013, en "Inteligencia social", Cuadernos Mente y Cerebro, p. 86).

"La arquitectura social del cerebro entrelaza los circuitos de la vía superior y de la vía inferior, dos sistemas que, en el cerebro intacto, operan en paralelo, como dos timones imprescindibles para navegar adecuadamente en el mundo social" (Goleman, 2006, p. 143). La vía superior equivale a la razón y la vía inferior a las emociones; es decir, un sano equilibrio entre la razón y las emociones tienen una incidencia directa en las relaciones sociales y por tanto en la salud y bienestar personal.

\subsection{Neuroplasticidad cerebral}

Uno de los descubrimientos más importantes en el mundo de la neurociencia señala que el cerebro cambia por la experiencia y por las interacciones sociales que tenemos. Los pensamientos crean reacciones químicas y sensaciones en el cuerpo. Los pensamientos conscientes que se repiten constantemente se convierten en inconscientes y generan patrones de comportamiento automático, lo que equivale a tener reacciones automáticas ante ciertos acontecimientos. Al igual que las emociones desencadenan reacciones automáticas, los pensamientos automáticos también generan reacciones químicas automáticas en el cuerpo.

La estructura cerebral de cada persona es única, producto de la herencia de nuestros padres y ancestros así como las experiencias que vivimos en nuestro entorno moldean a la vez nuestros circuitos neuronales; cuando aprendemos algo nuestro cerebro cambia:

Así pues, no sólo la estructura física del cerebro cambia ligeramente con la experiencia, sino que el aprendizaje modifica también sus características químicas. Aún hay que verificar si es algo permanente. De todos modos no hay 
duda de que para una adaptación óptima, no todo aprendizaje debería ser permanente. (Blakemore \& Firth, 2007, p. 198).

Es necesario también desaprender y reaprender para tener una adecuada adaptación en el mundo social.

La neuroplasticidad es la capacidad que tiene el cerebro de reorganizarse y de establecer nuevas conexiones sinápticas que le permiten aprender cosas nuevas y crear nuevas experiencias para actuar de diferente manera a la habitual si queremos conseguir diferentes resultados a los que estamos acostumbrados, si cambiamos la forma de hacer las cosas, nuestro cerebro cambiará y los resultados de nuestros actos también. Para "aprenhender", es decir, hacer nuestro el conocimiento, aplicarlo y repetirlo muchas veces a través de nuevas experiencias:

La "neuroplasticidad" del cerebro explica asimismo el papel que desempeñan las relaciones sociales en la remodelación de nuestro cerebro, lo que significa que las experiencias repetidas van esculpiendo su forma, su tamaño y el número de neuronas y de conexiones sinápticas. De este modo, la reiteración de un determinado registro permite que nuestras relaciones clave vayan moldeando gradualmente determinados circuitos neuronales. No es de extrañar por tanto que, sentirnos crónicamente maltratados o enfadados, o por el contrario emocionalmente cuidados por una persona con la que pasamos mucho tiempo a lo largo de los años, acabe remodelando los senderos neuronales de nuestro cerebro. Estos nuevos hallazgos ponen de relieve el impacto sutil y poderoso que sobre nosotros ejercen las relaciones. $Y$ aunque estas novedades pueden resultar desagradables, en el caso de que tiendan hacia lo negativo, también implican que el mundo social nos proporciona, en cualquier momento de nuestra vida una oportunidad de curación. Desde esta perspectiva, pues, el modo en que nos relacionamos cobra una importancia anteriormente insospechada. (Goleman, 2006, pp. 24-25).

Por ello, las relaciones sociales que entablamos tienen una importancia fundamental para nuestra salud y gracias al descubrimiento de la neuroplasticidad hay posibilidades de curación emocional y en las relaciones sociales, evitando las relaciones tóxicas y buscando tener relaciones sociales beneficiosas.

Los conocimientos obtenidos por los neurocientíficos han crecido exponencialmente en los últimos años: el cerebro humano es moldeado por las experiencias personales que se viven en sociedad y los pensamientos tienen una gran influencia en las emociones y éstas a su vez tienen una importancia fundamental en la salud psicofísica. Tenemos gracias a la neuroplasticidad la posibilidad de modificar nuestro cerebro voluntariamente y por lo tanto podemos mejorarnos a nosotros mismos a través de vivir nuevas experiencias y del aprendizaje. Este aprendizaje es posible gracias a la concentración focalizada y el desarrollo de la atención. "Cuando enseñamos a los niños habilidades específicas de la inteligencia, realmente afectamos la forma en que actúan su cerebro y su sistema nervioso influyendo tanto en su salud mental y física". (Shapiro, 2000, p. 23).

"La neurociencia ha descubierto que podemos moldear y dar forma a nuestro entramado neurológico con el mero hecho de concentrar nuestra atención de 
manera cotidiana en algo determinado" (Dispenza, 2010, p. 24); este descubrimiento es muy importante pero la educación no utiliza este nuevo conocimiento por ejemplo en su práctica cotidiana salvo excepciones en programas específicos.

"Cuando aprendes nueva información, por insignificante que sea, las pequeñas células cerebrales establecen nuevas conexiones entre ellas que cambian lo que eres." (Dispenza, 2010, p. 25); esta perspectiva es muy importante porque son las conexiones neuronales las que establecen nuestros comportamientos y son los pensamientos repetidos que crean las emociones que finalmente se reflejan en nuestra conducta en la vida social.

La neuroplasticidad otorga al cerebro la posibilidad de cambiar las conexiones entre las neuronas, lo que nos permite cambiar nuestro comportamiento y evolucionar. Los conocimientos que no se utilizan se pierden porque las neuronas se agrupan y reagrupan de manera continua. Esto nos da la posibilidad de lograr un desarrollo personal que puede repercutir en el desarrollo social aunque sea a pequeña escala.

La neuroplasticidad nos permite cambiar otorgándonos la capacidad de conseguir que el cerebro renuncie a su estructuración interna habitual y diseñe nuevos patrones y combinaciones:

...el hecho de romper los hábitos de pensamiento, actuación, sentimientos, percepción o comportamiento, es lo que nos permite ver el mundo ( $y$ a nosotros mismo) de una manera diferente. Y la mayor parte de este experimento de plasticidad es que tu cerebro cambia de forma permanente; activa un nuevo patrón de circuitos a nivel neurológico y eso hace que funcione de manera distinta. Has cambiado tu mente alterando el patrón típico cerebral y fortaleciendo las nuevas secuencias de conexiones celulares y de esta manera tú has cambiado también. Para nuestros propósitos, las palabras "cambio", "neuroplasticidad" y "evolución" tienen significados similares. (Dispenza, 2010, p. 34).

\subsection{Pensamiento y emoción, gestión emocional y pensamiento positivo}

Las emociones dependen de los pensamientos que habitualmente tenemos que se van adquiriendo por nuestra experiencia personal en el entorno. De la gestión de las emociones depende nuestro bienestar y salud. Las emociones cuando duran más tiempo del debido pueden ser perturbadoras, incluso destructivas.

La gestión emocional es la base para el bienestar psicofísico y la salud. Si hemos aprendido a tener ira, miedo, tristeza que son las emociones negativas podemos "desaprender" las emociones negativas y aprender las emociones positivas que generan salud y bienestar. Hay personas que tienen una gran capacidad para tener emociones que tienen una mayor duración que la mayoría de personas y en general experimentan más emociones negativas que positivas con lo cual perjudican su salud y necesitan un entrenamiento en la gestión emocional para tener más salud y ser más felices. Cuando constantemente sentimos emociones negativas éstas nos dan un estado de ánimo que en el caso de durar mucho tiempo puede generar un temperamento igualmente perjudicial para la salud y la armonía interior. Por el contrario las emociones positivas como la alegría y la compasión generan serenidad y felicidad. 
Los padres, madres, profesores podemos ayudar a nuestros hijos y/o alumnos a enfrentar y gestionar las emociones negativas y sustituir por emociones positivas que expresen afecto y éstas se desarrollan desde la más tierna infancia. Los pensamientos negativos pueden generar incluso lesiones físicas en el cerebro y los pensamientos positivos generan salud. He aquí un ejemplo de la interacción constante entre el pensamiento, las sensaciones que se grafican en el comportamiento y el estado de ánimo y salud de las personas:

Cuando el cuerpo responde a un pensamiento con una sensación, ésta desencadena una respuesta en el cerebro. El cerebro, que examina y evalúa sin cesar el estado del cuerpo, nota que éste se siente de determinada manera $y$, en respuesta a esa sensación corporal, genera pensamientos que provocan sus correspondientes mensajeros químicos de manera que comienzas a sentirte mal tal y como estás pensando. Los pensamientos generan sensaciones $\mathrm{y}$, acto seguido, las sensaciones generan pensamientos, lo que se repite en un círculo interminable. (Dispenza, 2010, p. 74).

Los pensamientos son poderosos porque influyen en nuestra salud, tienen la capacidad de alterar la bioquímica de nuestro cuerpo y con ello nuestra salud pues si son pensamientos agradables activan una sustancia llamada dopamina que nos hace sentir felices y si tenemos pensamientos tristes, negativos, de odio y rencor nuestro cuerpo produce neuropéptidos que afectan negativamente nuestra salud; de esta manera nuestros pensamientos están afectando de manera positiva o negativa nuestra salud.

"No debes olvidar que aquello en lo que piensas con más frecuencia determina lo que eres y en lo que te convertirás" (Dispenza, 2010, pp. 27-28); este es el enorme poder del pensamiento que afecta a nuestro cuerpo químicamente; de acuerdo a lo que pensamos producimos neutransmisores, péptidos y hormonas. Incluso cualquier recuerdo tiene componentes emocionales que tienen su respuesta química en nuestro organismo: “...y esos sentimientos son después lo que dan origen a nuestra forma de manifestar las actitudes, los comportamientos, las opiniones, los prejuicios, las creencias e incluso las percepciones. Nuestros sentimientos gobiernan nuestros pensamientos" (Dispenza, 2010, p. 414).

El pensamiento deja huellas tangibles en el cerebro a través de la representación mental; al respecto:

Desde el momento en que nuestra percepción se centra en una representación mental y el que se convierte en algo más real para nosotros que el mundo que nos rodea, comenzamos a crear nuevas conexiones en nuestro cerebro. La corteza prefrontal crea nuevas redes fuera del territorio conocido de nuestra personalidad para que el cerebro pueda almacenar, y después experimentar, la nueva información. De esta forma, el lóbulo frontal puede dejar un mapa de nuestra percepción consciente en el cerebro, almacenado como un nuevo recuerdo. Este proceso de almacenamiento y planificación proporciona evidencias físicas de que la mente ha experimentado un pensamiento; así pues, el pensamiento deja huellas tangibles en el cerebro humano (Dispenza, 2010, p. 519). 


\subsection{El desarrollo personal}

En su nivel más básico el Dr. Joe Dispenza señala que el desarrollo humano "es la capacidad de aprender y adquirir nuevos conocimientos" consideramos que el desarrollo humano integral tiene que ver con el hecho de crecer y evolucionar en sociedad como seres sociales que somos dotados de una realidad bio-psico-espiritual.

El ser humano es un ser social llamado al encuentro personal y con otras personas, y el encuentro más significativo lo realiza a través del amor, el matrimonio, la amistad y la cooperación en general que conlleva fines sociales. López (1994, p. 60) señala que: "todos procedemos de un encuentro y estamos llamado a fundar otros encuentros." Todos estamos llamados a ese encuentro que conlleva a un enriquecimiento mutuo a través del diálogo que nos conduce a un crecimiento y desarrollo personal. Pero este enriquecimiento y encuentro personal sólo es posible si nos hemos encontrado a nosotros mismos y si nos amamos en alguna medida para poder enriquecer con lo que somos a las personas con las cuales nos relacionamos y de igual manera ellos nos enriquecen con todo lo que son porque toda persona es única e irrepetible.

Para el encuentro es fundamental el respeto, que implica ayudar a otra persona a ser lo que está llamado a ser, es decir, colaborar con ella para que desarrolle sus capacidades y potencialidades al máximo para el bien común.

El hombre encuentra su pleno desarrollo personal en el amor porque lo enriquece en grado máximo y le otorga a su vida sentido y plenitud a través del encuentro personal. El amor implica incondicionalidad, entrega, fidelidad y compromiso que nos perfecciona como personas a través de la intimidad. Lo contrario al encuentro es el ensimismamiento, el egoísmo.

El desarrollo personal implica crecimiento y el crecimiento a su vez tiene en cuenta diferentes aspectos que podemos tener en cuenta a fin de tener una buena salud y bienestar psicofísico y espiritual Así tenemos que el ejercicio físico tiene una influencia positiva en la química cerebral según Blakemore \& Firth (2007) y genera beneficios en la salud mental ya que mejora el estado de ánimo de las personas, y es que la depresión es una de las enfermedades mentales más comunes en estas décadas y la prospección es que tendrá una mayor incidencia en los próximos años pues se calcula que en 2020 será la enfermedad más común del mundo occidental.

Las recomendaciones del Dr. Alonso, (2010) para tener una vida saludable señalan que: la respiración y la relajación física y mental favorece que nuestro sistema inmunitario y sistema nervioso se conserve saludable; también es muy importante la alimentación sana. La postura también es importante en la salud porque nos ayuda a sentirnos mejor. El perdón es otro de los temas importantes para la conservación de la salud de manera integral; este concepto engloba también la forma como percibimos la realidad y cómo la interpretamos desde nuestras experiencias; gracias al cambio de pensamientos y perspectiva podemos tener una visión diferente de las cosas y restaurar nuestras relaciones humanas claves para sentirnos a gusto con nosotros mismos y con nuestro entorno. Dormir al menos 7 horas también es fundamental para nuestro bienestar y salud. 
Para progresar en el cambio personal hacia el crecimiento, el bienestar y por lo tanto hacia la salud es fundamental el "repaso mental", es decir, que imaginemos lo que queremos lograr; ello nos ayuda a lograr las conexiones cerebrales adecuadas para lograr nuestro objetivo.

\subsection{Programa de habilidades sociales en Estados Unidos y el mundo anglófono}

Existen muchos programas de desarrollo de habilidades sociales y educación emocional diseñados por Mark Grenberg, y Lina Lantieri entre otros que trabajan en diversos ámbitos de la sociedad norteamericana: principalmente en las escuelas y en barrios con altos niveles de conflictividad social y que han obtenido un gran éxito. Desde 1994, la institución Collaborative for Academic, Social and Emotional Learning (CASEL) que se encuentra en la ciudad de Chicago está concitando la atención mundial sobre la importancia de estos temas para el desarrollo y maduración de los niños y que este aprendizaje emocional y social tiene un impacto en el desarrollo académico y la felicidad de los niños y jóvenes. Los fundadores que son psicólogos y psicopedagogos y filántropos fueron liderados por Daniel Goleman, Eileen Rockefeller Growald y Lina Lantieri.

\section{RESULTADOS}

Desarrollar programas similares en España y Latinoamérica que se adapten a las realidades sociales y que se incluyan en la educación no formal a través de actividades extraescolares, asociaciones, centros de ocio y tiempo libre juvenil que aplique los conocimientos esbozados de la neuroplasticiad para desde allí utilizar el método de investigación-acción participativa o desde el método experimental: con dos grupos, uno que trabaje talleres de desarrollo socio-emocional y un grupo de control a fin de analizar los resultados obtenidos.

Los programas podrían ser de conocimiento personal, comunicación no verbal, resolución de conflictos, desarrollo de la empatía y la compasión, comunicación interpersonal, autoestima, liderazgo, pensamiento positivo, desarrollo de la atención y la concentración, relajación mental entre otros temas de importancia en el desarrollo de las relaciones sociales.

\section{CONCLUSIONES Y DISCUSIÓN}

Los descubrimientos de la neuroplasticidad cerebral tienen aportes importantes para el desarrollo del potencial humano y se pueden usar en beneficio del desarrollo personal de los jóvenes y de las personas en general independientemente de su etapa de desarrollo evolutivo. Los pensamientos tienen una importancia fundamental en el desencadenamiento de las emociones y las sensaciones que tienen una implicancia en el bienestar y salud psicofísica y emocional.

El modelado de nuevas experiencias y el aprendizaje de programas de habilidades socioemocionales tienen un impacto favorable entre las personas que participan en estos programas y además tienen una incidencia favorable en las sociedades en la que se aplica porque se da una disminución de la violencia en ellas. 
La inteligencia social es muy importante para la salud y el desarrollo y crecimiento personal pues a través de las relaciones positivas crecemos como personas. En última instancia la inteligencia social es aprender a conocer y amar a las personas así como aceptarnos a nosotros mismos y los demás para gestionar adecuadamente las emociones que nos generen bienestar. Asimismo el desarrollo emocional y social tiene una relación directa en el desarrollo cognitivo de las personas.

\section{REFERENCIAS}

Alonso, M. (2010). Reinventarse: tu segunda oportunidad. Madrid: Plataforma Editorial.

Bandura, A. y Richard Walters, (1988). Aprendizaje social y desarrollo de la personalidad. Traducción de Angel Riviére. Alianza Editorial.

Blakemore, S-J. y Uta Firth, (2007). ¿Cómo aprende el cerebro humano? Barcelona: Hurope S.L.

Dispenza, J. (2010). Desarrolla tu cerebro. Madrid: La esfera de los libros.

Ekman, P. (2003) ¿Qué dice ese gesto? Barcelona: RBA Libros.

Goleman, D. (2003). Emociones destructivas: Cómo entenderlas y superarlas. Barcelona: Editorial Kairós.

Goleman, P. (2006). Inteligencia social: La nueva ciencia de las relaciones humanas. Editorial Kairós.

Greenspan, S. y Nancy Thordike, (1997). Las primeras emociones. Barcelona: Editorial Paidós.

Lantieri, L., (2014). Las emociones van a la escuela. Consultado el 14 de junio de 2014. Recuperado de

http://www.lindalantieri.org/documents/NationalGeographicarticleElthroughSEL Spanish.pdf

López-Quintas, A., (1994). El amor humano, su sentido y su alcance. Madrid: Edibesa.

Marschall, J. (2013). Inteligencia social en Cuadernos Mente y cerebro, 5, 82-96.

Nagel, I. (2013). Vejez personal en Cuadernos Mente y cerebro, 5, 54-60.

Shapiro, L. (2000). La salud emocional de los niños: Cómo los padres pueden evitar los problemas emocionales de sus hijos antes que se desarrollen. Madrid: Editorial EDAF. 